\title{
Criminologie
}

\section{Les transformations de la pénalité}

\section{Philippe Robert}

Volume 40, numéro 2, automne 2007

Peines et pénalité au Canada. Autour des travaux de Pierre Landreville

URI : https://id.erudit.org/iderudit/016851ar

DOI : https://doi.org/10.7202/016851ar

Aller au sommaire du numéro

Éditeur(s)

Les Presses de l'Université de Montréal

ISSN

0316-0041 (imprimé)

1492-1367 (numérique)

Découvrir la revue

Citer cet article

Robert, P. (2007). Les transformations de la pénalité. Criminologie, 40(2), 53-66. https://doi.org/10.7202/016851ar

\section{Résumé de l'article}

S'attachant à l'un des thèmes favoris du professeur Landreville, la peine, l'auteur montre l'intérêt de le mettre en perspective dans le long terme historique.

Il commence par expliquer comment l'émergence décisive de la pénalité est liée à celle de l'étatisation de nos sociétés.

Puis il étudie la liaison entre différentes formes d’État et différents régimes de pénalités.

Enfin, il s'attache, à partir de données provenant d'un pays de l'Europe occidentale, la France, à scruter les recompositions actuelles de la pénalité, dans un État devenu moins social et plus libéral.
Ce document est protégé par la loi sur le droit d'auteur. L'utilisation des services d'Érudit (y compris la reproduction) est assujettie à sa politique d'utilisation que vous pouvez consulter en ligne.

https://apropos.erudit.org/fr/usagers/politique-dutilisation/ 


\title{
Les transformations de la pénalité
}

\author{
Philippe Robert
}

Directeur de recherches émérite

Centre de recherches sociologiques sur le droit et les institutions pénales (CESDIP), CNRS probert@gern-cnrs.com

RÉSUMÉ - S'attachant à l'un des thèmes favoris du professeur Landreville, la peine, l'auteur montre l'intérêt de le mettre en perspective dans le long terme historique.

Il commence par expliquer comment l'émergence décisive de la pénalité est liée à celle de l'étatisation de nos sociétés.

Puis il étudie la liaison entre différentes formes d'État et différents régimes de pénalités.

Enfin, il s'attache, à partir de données provenant d'un pays de l'Europe occidentale, la France, à scruter les recompositions actuelles de la pénalité, dans un État devenu moins social et plus libéral.

ABSTRACT • Focussing on one of Prof. Landreville favourite themes, punishment, this article shows the importance of placing it in a very long term historical perspective.

The first section explains how the emergence of punishment as a social system is linked with the emergence of the modern State.

The second section analyses correlations between different forms of State and various types of penalties.

Finally, using recent data from a west-European country, France, the article examines current re-casting of punishment in an increasingly liberal and less a welfare State.

\section{Introduction}

Pierre et moi sommes amis depuis mon premier séjour à l'Université de Montréal en 1969, depuis sa première sabbatique passée en bonne partie dans mon centre (alors tout nouveau).

Tout au long de ce grand tiers de siècle, nous sommes restés d'autant plus proches que nos points de vue et nos manières d'aborder les problèmes convergent assez spontanément. 
Cette convergence est d'autant plus remarquable que nos thèmes de recherche ont été, le plus souvent, assez différents. Si nous avons partagé un intérêt commun pour la sociologie de la création de la norme pénale (Landreville, 1991 ; Lebeuf et Landreville, 1988) - ce qui nous a notamment conduits à participer ensemble en 1991 à un séminaire que j'organisais à Oñati sur la création de la loi (Robert, 1991) -, les sujets qui ont le plus retenu l'attention de Pierre - les coûts sociaux de l'intervention pénale (Pires, Landreville et Blankevoort, 1981), la détermination de la sentence (Pires et Landreville, 1985), la criminalisation des problèmes sociaux, notamment de l'itinérance (Laberge, Landreville, Morin et al., 1998), surtout la peine - ne sont pas ceux que j'ai le plus travaillés.

Quand nous nous sommes rencontrés pour la première fois, à la fin de la décennie 1960, il terminait sa thèse La prédiction de la gravité de l'agir délinquant. Ensuite, la peine est restée le point d'ancrage essentiel de ses recherches, même s'il en a considérablement élargi les frontières en s'intéressant à l'évaluation des impacts de l'emprisonnement (Landreville, 1982), également aux alternatives (Landreville et al., 1986) et substituts à l'emprisonnement (Landreville, 1987; 1999). De fil en aiguille, son investissement s'est étendu à l'ensemble des politiques et pratiques pénales dans une optique de plus en plus internationale. C'est ainsi qu'il a publié dans un manuel collectif européen un article remarqué sur le point (très controversé) de savoir s'il existe un modèle pénal étasunien et s'il sert de référence dans d'autres parties du monde (Landreville, 2002).

Il n'est donc pas surprenant que les promoteurs de ce Liber amicorum m'aient assigné un thème qui touche à la pénalité.

Je parlerai seulement des peines et non pas ${ }^{1}$ du thème - trop large pour une brève contribution - des politiques pénales qui les utilisent ou les mettent en œuvre. Reste à décider comment l'aborder.

S'essayer à décrire en quoi la peine a changé ces dernières décennies, d'autres l'ont déjà entrepris. Pierre, lui-même, s'y est récemment attaché dans une perspective comparative États-Unis-Canada-Europe occidentale (Landreville, 2006).

Je vais placer ma réflexion dans une perspective de plus long terme. Quand on étudie les transformations de la pénalité sur le moyen terme de quelques décennies, on est toujours tenté de poser au moins impli- 
citement le régime d'hier comme «normal» et de voir ses altérations récentes comme des anomalies. Adosser cette réflexion à une mise en perspective sur le long et même le très long terme permet d'insérer les mutations contemporaines dans une histoire. Pour diminuer les risques d'anachronisme, pareille opération suppose de commencer par situer la peine dans une conceptualisation sociologique.

\section{La peine corollaire de l'étatisation des sociétés}

Pour un sociologue, Les Règles de la méthode ${ }^{2}$ constituent un bon point de départ à l'étude de la peine: Durkheim, on s'en souvient, y appelle crime ce qui est sanctionné - il serait plus exact de dire sanctionnable - par une peine. J'ai beaucoup insisté moi-même sur la nécessité pour les sociologues de repartir de cette définition provisoire et de construire leur programme de recherche à partir d'elle (Robert, 2005).

Mais qu'est-ce qu'une peine (Robert, 1996; 1998; 2005)? Pour répondre, rien ne vaut de se demander ce qui se passe dans les sociétés qui l'ignorent. Poser cette question suppose évidemment de renoncer au point de vue anachronique selon lequel toute société humaine connaîtrait un droit pénal.

Dans les sociétés «segmentaires» (Evans-Pritchard et Fortes, 1964 [1940]) où l'étatisation du politique reste absente ou en tout cas faible, on repère bien, éventuellement, quelques rares incriminations (délits publics) qui sanctionnent par la mise à mort ou par le bannissement, en tout cas par l'exclusion, des atteintes concernant les relations de la Cité avec ses divinités, ou la conduite de la guerre, mais cela ne suffit pas pour faire un pénal. Les dommages entre clans ou entre familles se règlent, pour l'essentiel, par un autre procédé, la vengeance.

Marcel Mauss (1896) l'avait déjà suggéré, des travaux anthropologiques plus récents (Courtois, 1984) le confirment, le visage originaire de la vengeance est tout à l'inverse d'un enchaînement répétitif de représailles sans fin, d'une passion vindicative. Il s'agit pour le clan ou la famille victime d'infliger à celui dont provient le tort un dommage équivalent et, pour celui-ci, de l'accepter comme tel. Cette rétorsion peut intervenir en nature, mais aussi par une compensation plus ou moins rigidement tarifée et dont les modalités d'adjudication peuvent

2. Durkheim, 1895 (p. 35 et accessoirement 42 de la quatorzième édition). 
être plus ou moins judiciarisées. L'acquittement de cette dette remet les deux parties dans l'état d'équilibre antérieur de sorte qu'il leur devient alors possible de reprendre leurs relations sociales.

L'étatisation du politique fait basculer dans un autre système de contrôle de la violence. Une société sans État la maîtrise en veillant au maintien et au rétablissement, par le jeu du vindicatoire, d'un (relatif) équilibre de forces entre les clans. Une société étatisée se propose de la contenir par l'instauration d'un déséquilibre - entre le souverain qui inflige la peine et l'individu qui la subit - auquel rien ni personne ne résiste et que nul ne peut concurrencer. La peine est en rupture avec la vengeance, non pas comme la civilisation par rapport à la barbarie, mais parce qu'il s'agit d'une logique différente de contention de la violence.

Elle menace les auteurs de certains comportements. Parmi ces derniers, les uns ont effectivement causé du tort à une victime particulière, d'autres sont seulement réputés dangereux, attentatoires à un intérêt commun ou à l'autorité publique. Mais, dans un cas comme dans l'autre, le pénal procède toujours en organisant un affrontement entre le simple particulier fauteur de troubles et la puissance publique. Par son action, voire son omission, quelquefois son mode de vie, le criminel, proclamet-il, s'est attaqué à la souveraineté. C'est pourquoi la sanction prend la couleur d'un châtiment. Il s'agit moins de réparer le tort que de punir l'audace du contrevenant et, par là, de rappeler la différence insurmontable qui sépare le souverain du particulier.

Le mécanisme pénal implique une puissance publique et même une puissance publique suffisamment autonome, suffisamment exhaussée au-dessus de l'ordinaire des relations sociales, pour avoir la force de creuser cette différence entre une partie publique souveraine et une autre simplement privée. Autrement dit, l'établissement d'une véritable économie pénale suppose une étatisation. Le politique acquiert alors une stabilité et une autonomie que ne possédait pas la magistrature civique toujours prête à retomber dans l'ordinaire des relations sociales. En identifiant un espace "public» propre au politique, en l'institutionnalisant, l'étatisation fait apparaître par contrecoup un espace privé, la «société civile». Plus que la concentration de puissance qui fascine, c'est cette mise à part d'une zone publique qui signe l'étatisation d'une société.

L'établissement d'une réelle économie pénale suppose que la puissance publique monopolise, en force et en légitimité, assez de ressources pour opérer un véritable coup de force: la publicisation de délits privés. 
Au motif que l'agression ou la prédation constitue un défi à sa prétention à se constituer en gardien général de la paix - autrement dit à sa revendication de monopoleur de la violence à l'intérieur d'un territoire -, elle se substitue à la victime dans le rôle de l'offensé. Avant même de constituer une branche du droit, le pénal est une figure de procès où l'autorité publique joue deux rôles, non seulement celui du juge, mais encore celui du plaignant. Plus le politique est étatisé, plus il a la capacité de publiciser ainsi un nombre important de délits privés. En même temps, l'exhaussement du politique au-dessus de l'ordinaire des relations sociales, son étatisation, entraîne une démultiplication de l'antique noyau de délits publics, ceux sans victime directe, qui croissent à proportion de l'extension des compétences abandonnées à ou conquises par la puissance publique.

Le propre de ce modèle de contention de la violence est justement la prétention à traiter les délits privés publicisés de la même manière que les délits publics, en menaçant d'un châtiment, et non d'une réparation, celui qui s'en rendrait coupable. Toute l'attention se concentre sur ce dernier: au lieu de chercher à remettre sur le même plan deux parties analogues, on souligne l'incommensurabilité entre ce délinquant privé et le souverain qui le poursuit. Pour cela, on emprunte la sanction du vieux délit public: la peine remplace ces règlements qui assuraient l'indemnisation de la victime. Là se situe le sens premier et toujours fondamental de la peine, quels que soient les compromis ou les atténuations que révèlent les historicités concrètes.

Observer dans la profondeur diachronique la liaison peine-État est cependant malaisé. D'abord, l'histoire de l'État est complexe: non seulement elle est plutôt processus lent et obscur que soudaine irruption d'un modèle tout armé - il est plus prudent de parler de sociétés faiblement ou fortement étatisées que d'opposer brutalement des formations sociales avec et sans État -, mais encore elle n'a rien de linéaire. Si dans la Rome antique la société s'est lentement étatisée dans le passage au principat et, plus encore, au dominat, le premier féodalisme des sociétés européennes ne l'est plus guère. Il faudra attendre le passage au féodalisme centralisé et surtout à l'absolutisme royal pour voir apparaître de nouveau une étatisation progressive du politique. Ajoutons encore que l'État a déjà revêtu des formes bien diverses et qu'on en verra peut-être encore d'autres variétés: pour généralisée que soit actuellement la figure de l'État-nation, ce n'est pas pour autant la seule modalité possible d'État. 
Autre difficulté, la corrélation étatisation-pénalisation n'est souvent pas immédiatement lisible dans la concrétude historique. Du coup, ce versant important du processus d'étatisation n'est pas toujours clairement pris en compte par les historiens de l'État (Cpdt Rousseaux et Lévy, 1997). Pour autant, identifier le pénal aux sociétés segmentaires ne résiste ni aux analyses de Max Weber (1986 [1911-1913]), ni surtout aux travaux anthropologiques (Le Roy et Trotha, 1993). Il apparait, au contraire, fortement lié à la modernité étatique.

\section{Formes d'État et régimes successifs de la peine}

Pour autant, ce serait pousser trop loin le souci de modélisation que d'imaginer une peine immobile: les différents avatars de l'étatisation lui ont fait subir de multiples mutations.

Nous disposons de plusieurs théorisations historicistes de la peine. Tout comme celle d'Ignatieff (1978), celle de Foucault (1975) s'attache à un moment précis de l'histoire de la pénalité. Il s'agit pour lui d'expliquer l'improbable émergence de la prison, à la charnière du XvIII et du $\mathrm{XIX}^{\mathrm{e}}$ siècle, comme moyen de distinguer «illégalismes de droit», apanage des dominants, et «illégalismes de fait», ceux du populaire, en faisant l'économie d'une référence à l'État: Foucault disperse le pouvoir dans l'ensemble des relations sociales avec le risque, bien "postmoderne», d'une scène finalement déserte.

Celle de Sellin (1976) - inspirée de Radbruch (1950) - défend l'idée, très linéaire, selon laquelle l'esclavage antique aurait constitué la matrice récurrente de la peine et qu'elle se retrouverait notamment dans le travail forcé de la société moderne.

La théorie la plus élaborée est finalement la plus ancienne: Rusche envisage l'évolution de la peine dans l'Ouest européen et en Amérique du Nord comme liée aux mutations du rapport de l'homme à la situation de travail (Rusche et Kirchheimer, 1939). Pour riche qu'elle soit, cette vaste fresque accorde peut-être trop peu d'importance à la rupture de logique entre vindicatoire et pénal (en début de processus), aussi aux avatars ultérieurs de l'étatisation du politique, sauf éventuellement in fine avec la considération au moins implicite de l'État social.

Si aucune de ces grandes reconstitutions ne suffit à rendre compte des changements de la pénalité, elles encouragent cependant à mettre la peine dans une perspective historique, même s'il faut aller chercher 
dans les mutations de l'étatisation le meilleur indicateur pour comprendre cette histoire de la peine.

À l'aube de l'État moderne, les historiens ont souvent relevé une brutale pénalisation de la justice que traduit un acharnement sur le corps du coupable, comme s'il s'agissait d'instiller de l'obéissance, ou au moins du respect, d'affirmer le caractère irrésistible de la prééminence royale. Il est vrai que les justices extra-étatiques d'antan - urbaines, seigneuriales ou ecclésiastiques - étaient mal armées pour faire face à la montée de l'errance de franges rurales appauvries qui se pressaient aux alentours des villes. Cependant, l'étatisation mettra longtemps à se faire accepter en profondeur, surtout ses tours de vis fiscaux; et chaque «émotion» populaire suscitera en réponse un hoquet répressif sanglant, mais bref. Mais c'est particulièrement la répression de la sorcellerie qui rythmera, notamment du milieu du Xvi ${ }^{\mathrm{e}}$ à celui du XvII ${ }^{\mathrm{e}}$ siècle, cette pénalisation féroce de la justice et cet apprentissage de l'obéissance.

Cependant, quand le respect s'est suffisamment insinué le long des hiérarchies sociales, on a assisté à une accalmie sur le front de la peine. La punition s'est réduite pour l'essentiel à un couple déséquilibré: quelques exécutions spectaculaires - qui réaffirmaient le caractère fondamentalement inexpiable du défi à la souveraineté étatique - et un grand nombre d'éloignements qui se bornaient à rejeter à bon compte le coupable, soit qu'on le bannisse, soit qu'il prévienne la sanction en prenant le large. C'est le modèle-type de la pénalité de l'Ancien Régime européen dans sa dernière étape. Le contrôle des franges marginales, de ceux qui erraient, a mobilisé une diversité de prises en charge: si le «grand renfermement» n'a peut-être pas joué un rôle aussi important qu'on l'a supposé un temps, il ne faut pas sous-estimer, en revanche, la prise en charge par l'appareil monarchique le plus solide, l'armée: soit qu'on enrôle de force (la presse) le «sans-aveu», soit qu'on l'envoie aux galères, soit encore qu'on le déporte outre-mer avec la première expansion coloniale. Au fond, c'est encore d'éloignement qu'il s'agit.

Mais la mise à mort va devenir de plus en plus difficile quand le fondement de la souveraineté redescendra sur terre, que son lien avec le sacré s'affaiblira. C'est pourquoi Beccaria (1966 [1764]) est abolitionniste, parfois aussi les codes de la fin du XVIII siècle et c'est de peu que la Constituante française refuse finalement de supprimer, comme le proposaient les Comités réunis, la peine capitale. Par ailleurs, toute une série de raisons rendent l'éloignement de moins en moins aisé: l'unification $\mathrm{du}$ territoire national contredit la pratique antérieure du bannissement 
hors des limites d'une seule juridiction; l'adoption de la fiction du contrat social comme base nouvelle de la souveraineté ne facilite pas l'expulsion des citoyens.

On peut voir un signe de cette réorganisation dans les entreprises pour ériger le travail forcé en sanction pénale type au moment où la liberté du travail prenait figure de norme fondamentale de l'organisation sociale. Pour la première fois, du moins à grande échelle, s'avère nécessaire une prise en charge des punis dans la durée. Bagne, pénitencier ou prison, c'est cette perte de liberté - avec généralement une obligation de travail - qui devient la peine-type de l'État libéral du XIX ${ }^{e}$ siècle. Dès lors, une répression, plus régulière mais plus extensive, peut succéder à celle, rare même si parfois spectaculaire, de l'Ancien Régime.

Une autre recomposition majeure de la pénalité se fera jour avec les prémisses de l'État social. Il ne paraîtra plus utile d'enfermer effectivement l'ordinaire des coupables: pour eux, la menace - sursis, sentence suspendue - suffira quand l'on n'opte pas pour une amende. L'emprisonnement entamera alors un long cycle baissier ${ }^{3}$ qui ne se renversera que dans les dernières décennies du $\mathrm{xx}^{\mathrm{e}}$ siècle. En même temps, cependant, le récidiviste, lui, paraît dangereux. S'il semble irrécupérable, rôdent des fantasmes d'élimination qui se concrétisent plus ou moins ouvertement selon les pays. Qu'il semble, au contraire, rééducable - le jeune prolétaire -, on entreprend de jeter, par la "prévention» et les mesures éducatives, un pont entre la peine et la politique sociale. L'économie de la peine se segmente.

\section{Recompositions contemporaines de la pénalité}

Pour tenter de voir ce qui a changé, je n'utiliserai pas le cas des ÉtatsUnis qui est trop extrême et donc trop atypique ${ }^{4}$; je me bornerai à montrer comment le régime des peines a changé en un quart de siècle dans mon propre pays avec cet avantage qu'on pourrait dire à peu près la même chose de ses voisins européens.

3. Pour la France, voir Barré (1986); pour l'Angleterre, Radzinowicz (1999: 86-89).

4. Même si bon nombre d'essayistes y voient - pour s'en féliciter ou le déplorer - le modèle vers lequel les autres États de l'Ancien et du Nouveau Monde doivent fatalement s'acheminer. Au demeurant, Young (1999) raillait l'américanomanie pénale des élites politiques européennes quand il écrivait qu'aller chercher des recettes pour contrôler le crime aux États-Unis revient à peu près à étudier les droits des femmes en Arabie saoudite. 
Au milieu des années 1970, le détenu type était un voleur qui restait peu de temps en prison; maintenant, c'est un violeur, un meurtrier ou un trafiquant de drogue qui y reste longtemps ${ }^{5}$. On a fait le choix de consacrer surtout cette ressource aux violences et aux affaires d'ordre public - drogue et immigration irrégulière. On a aussi opté pour un allongement considérable de la durée des peines prononcées: il n'entre pas davantage des gens en prison, mais ils y restent beaucoup plus longtemps. Le résultat est clair: le voleur ordinaire y a beaucoup moins sa place; quant à l'agresseur, la prison s'occupe surtout de l'auteur de grands crimes - notamment sexuels - bien plus que de l'arracheur de portable ou du membre de bande bagarreur.

TABLEA U 1

Incarcérés au $\mathbf{1}^{\text {er }}$ janvier d'après la sorte d'infraction

\begin{tabular}{|l|c|c|c|c|}
\hline \multirow{2}{*}{ Détenus au 1' janvier } & \multicolumn{2}{|c|}{$01 / 01 / 1975$} & \multicolumn{2}{c|}{$01 / 01 / 2005$} \\
\cline { 2 - 5 } & Nombre & $\%$ & Nombre & $\%$ \\
\hline Atteintes volontaires contre les personnes & 3011 & 23,2 & 17570 & 48,4 \\
\hline Atteintes aux biens & 8270 & 63,8 & 6010 & 16,5 \\
\hline Atteintes économiques et financières & 311 & 2,4 & 2576 & 7,1 \\
\hline Infractions d'imprudence et routières & 93 & 0,7 & 2005 & 5,5 \\
\hline Stupéfiants, immigration irrégulière & 116 & 0,9 & 6150 & 16,9 \\
\hline Autres & 1171 & 9,0 & 2019 & 5,5 \\
\hline Ensemble & 12972 & 100,0 & 36330 & 100,0 \\
\hline
\end{tabular}

Source: Robert, 2002 actualisé.

Pour l'auteur des méfaits qui interviennent le plus dans l'insécurité quotidienne des citoyens - vols, cambriolages, dégradations, petites agressions -, on a surtout développé des sanctions dites intermédiaires : ni l'incarcération, ni l'amende - que les juges cantonnent de plus en plus à la circulation routière -, mais le sursis ou des peines en milieu ouvert comme la mise à l'épreuve ou le travail d'intérêt général.

5.... voire un immigrant clandestin qui y reste peu de temps, mais y revient souvent. 
TABLEAU 2

Peines (crimes, délits, contraventions de $5^{\mathrm{e}}$ classe) en $\%$

\begin{tabular}{|l|c|c|c|c|}
\hline \multirow{2}{*}{ Peines } & \multicolumn{2}{|c|}{1975} & \multicolumn{2}{c|}{2005} \\
\cline { 2 - 5 } & Nombre & $\%$ & Nombre & $\%$ \\
\hline Emprisonnements & 96070 & 17,8 & 114164 & 19,3 \\
\hline Amendes & 303569 & 56,2 & 198402 & 33,5 \\
\hline Peines intermédiaires & 140639 & 26,0 & 279932 & 47,2 \\
\hline Ensemble & 540278 & 100,0 & 592498 & 100,0 \\
\hline
\end{tabular}

Source: Robert, 2002 actualisé.

Grand changement: la justice a trouvé une pénalité spécifique pour la «petite» délinquance. Ces peines intermédiaires sont, quantitativement, le gagnant du dernier quart de siècle. Et ce constat paraît d'autant plus net que l'on pourrait ajouter à ces peines intermédiaires les 461203 mesures alternatives aux poursuites ou compositions pénales que le ministère public a utilisées en 2005 comme formes de traitement extrajudiciaire de la petite délinquance ${ }^{6}$.

Toutefois, le triomphe des peines intermédiaires est un peu en trompe-l'œil. Depuis le milieu des années 1980, l'allongement ininterrompu de la durée des séjours en prison a conduit à accorder à cette dernière une priorité budgétaire absolue ${ }^{7}$. Du coup, les dispositifs qui gèrent les peines intermédiaires sont très fragiles. D'une part, les délais de mise à exécution - cette face rarement étudiée de la justice - empêchent de prendre les condamnés en charge dès que la sentence est devenue exécutoire. D'autre part, la mesure manque souvent de substance faute de moyens, notamment quand des agents, en trop petit nombre, doivent gérer chacun un nombre excessif de cas (Chauvenet $e t$ al., 1999).

\section{Au total:}

- un retour en force de l'emprisonnement surtout marqué par un allongement immodéré de la durée des séjours et par une concentration sur quelques délinquances très ciblées;

- une impressionnante poussée quantitative des peines intermédiaires sans, toutefois, une croissance équivalente des moyens de mise en œuvre.

6. Soit presque le tiers $(31,5 \%)$ de la matière première «utile» parvenant au ministère public (après déduction des affaires que la police n'a pas été capable d'élucider ou de celles qui ne sont pas pénalement caractérisées). Voir Justice (2006. 14).

7. Qui n’a pas empêché d'ailleurs un état des prisons régulièrement dénoncé. 
Même si les proportions varient d'un cas à l'autre et si l'hyper déchaînement répressif étasunien reste un cas à part qui demande des analyses spécifiques, la double conclusion qui s'évince de cette observation monographique s'extrapole assez bien quand on élargit la focale au delà du seul cas français.

Écartelé entre deux priorités largement contradictoires, la demande de sécurité quotidienne de ses citoyens et le contrôle de flux internationaux de personnes, de marchandises, d'argent et d'informations (Robert, 2000), l'État a largement préféré celui-ci à celle-là.

Certaines de ces opérations consomment surtout des procédures administratives, du Renseignement et de la coopération policière internationale - ainsi du contrôle des mouvements de blanchissement de l'argent sale ou de «noircissement» de l'argent légal, ou encore celui des circuits terroristes. En revanche, les tentatives pour maîtriser l'immigration illégale ou l'importation de drogues prohibées sont grandes consommatrices de ressources pénales. Au total, l'ordre public constitue la vraie priorité de ce système de peines.

En revanche, les gros appareils policier et judiciaire appréhendent difficilement la délinquance qui constitue un risque ordinaire pour les citoyens - vols, cambriolages, dégradations, petite violence prédatrice -, soit qu'elle leur est rarement «renvoyée» par les victimes (dans le cas de la violence de basse intensité), soit qu'elle n'est pratiquement jamais élucidée par la police (dans le cas de la délinquance d'appropriation), et ils ne lui consacrent pas une grande priorité de fait.

On s'efforce de donner le change et de satisfaire les préoccupations sécuritaires en frappant de manière de plus en plus obsessionnelle les cas (heureusement peu répandus) de violence spectaculaire, notamment sexuelle. On produit tout simplement un décalage entre une réponse pénale qui s'épuise à frapper toujours plus fort la grande violence et les atteintes à l'ordre public, et une victimation de petite violence et de prédation - et des désordres - en miettes (Robert, 1999) qu'on ne parvient guère à saisir et à prendre en charge.

D'où probablement le retour de la victime sur le devant de la scène, une sérieuse entorse à un processus de pénalisation qui, depuis son origine, tend à la déposséder plus ou moins totalement de son affaire. Ici, la logique punitive du pénal est sévèrement mise en cause; l'affrontement entre l'État et le délinquant perd de son exclusivité et l'on assiste à un regain d'intérêt pour l'affrontement entre l'auteur et la victime. Si la pénalisation ne semble plus capable d'assurer la sécurité des personnes 
et de leurs biens, alors les solutions vindicatoires d'indemnisation reprennent de leur lustre. Ne sommes-nous pas devant des États qui ont du mal à persuader leurs citoyens de leur capacité à assurer la sûreté publique? Ils peuvent certes tenter de se sortir de ce mauvais pas par des politiques sécuritaires qui prennent appui sur les fractions les plus insécures de la société. Mais il est aussi possible de caresser les victimes dans le sens du poil en s'intéressant à leur sort. Seulement, le processus répressif s'avère mal armé pour déployer l'intérêt qu'on veut leur prodiguer. Aussi bien, est-ce en dehors du procès pénal que se sont développés les mécanismes d'aide aux victimes et d'indemnisation. Mais ils ne représentent guère plus qu'une goutte d'eau dans la mer des victimations... outre que les victimes réclament souvent la symbolique pénale, la condamnation du coupable.

On peut certes lire la trace d'un retour vers un État plus libéral et moins social. Un État libéral recourt toujours à la répression pénale davantage qu'un État social ${ }^{8}$ dans la mesure où il dispose de moins de moyens alternatifs pour se faire entendre de ses citoyens et bénéficier de leur loyauté et de leur obéissance à ses normes. Pour autant, nous ne sommes pas revenus au régime de peines du premier $\mathrm{XIX}^{\mathrm{e}}$ siècle, au couple pénitencier-bagne; nous ne sommes pas non plus revenus au même État libéral qu'alors. À vrai dire, la dimension providentielle ou sociale de l'État actuel demeure importante et, en Europe continentale de l'Ouest, elle résiste fortement à l'érosion. Nous nous trouvons plutôt devant un mixte libéral-social dont le régime de peines est en quelque sorte original ${ }^{10}$.

En même temps, il s'agit d'un État affaibli par la concurrence que livrent à son pôle d'ancrage, la nation, et la déspatialisation relative de

8. Le terme souvent utilisé d'État-providence est moins heureux que celui, proposé par Castel (1995), d'État social.

9. En ce sens, les crispations répressives - l'avalanche incessante de nouvelles lois pénales, l'exaspération devant la récidive - traduisent peut-être surtout un secret désarroi (devant la perte de crédit du politique) que l'on tente de masquer derrière de mâles postures.

10. Même si les États de l'Ouest européen sont tentés de se focaliser de plus en plus sur les ressources répressives (par exemple Duprez et Hebberecht, 2001), il n'en reste pas moins que leurs politiques de sécurité comprennent également des programmes de prévention (même si souvent rabougris dans des postures seulement défensives) et des politiques sociales (même si leur effectivité semble trop limitée). Ce sont là des dispositifs typiques d'États sociaux. Quant au régime de la pénalité lui-même, la place des peines intermédiaires témoigne bien de la résistance de formules inventées avec l'État social (rappelons que le sursis et la libération conditionnelle ont été les mesures phares de la recomposition intervenue dans les décennies précédant la Première Guerre mondiale). 
la sphère économique (et surtout financière) et les constructions supranationales comme l'Union européenne.

$\mathrm{Au}$ total, il est donc possible que le régime des peines que nous observons actuellement soit aussi instable que l'État qui le génère.

\section{Références}

Barre, M. D. (1986). 130 années de statistiques pénitentiaires en France. Déviance et Société, 10 (2), 107-128.

Beccaria, C. (1966 [1764]). Traité des délits et des peines. Paris: Cujas.

Castel, R. (1995). Les métamorphoses de la question sociale: une chronique du salariat. Paris: Fayard.

Chauvenet, A., Gorgeon, C., Mouhanna, C., \& Orlic, F. (1999). Contraintes et possibles: les pratiques d'exécution des mesures en milieu ouvert. Paris: Acadie. Centre de sociologie des organisations, Centre d'études des mouvements sociaux.

Courtois, G. (Dir.) (1984). La vengeance, études d'ethnologie, d'bistoire et de philosophie. Paris: Cujas.

Duprez, D., \& Hebberecht, P. (Dir.) (2001). Les politiques de sécurité et de prévention en Europe. Déviance et Société, 25 (4).

Durkheim, É. (1895). Les règles de la méthode sociologique. Paris: Félix Alcan.

Evans-Pritchard, E. E., \& Fortes, M. (Dir.). (1964 [1940]). Systèmes politiques africains. Paris: PUF.

Foucault, M. (1975). Surveiller et punir. La naissance de la prison. Paris: Gallimard.

Ignatieff, M. (1978). A Just Measure of Pain: the Penitentiary in the Industrial Revolution, 1750-1850. New York: Pantheon.

Justice (2006). Les Chiffres-clés de la Justice. Paris: Ministère de la Justice.

Laberge, D., Landreville, P., Morin, D., \& Casavant, L. (1998). Le rôle de la prison dans la production de l'itinérance. Montréal: UQAM, CRI.

Landreville, P. (1982). La récidive dans l'évaluation des mesures pénales. Déviance et Société, 6 (4), 375-388.

Landreville, P. (1987). Surveiller et punir: l'assignation à domicile sous surveillance électronique. Déviance et Société, 11 (3), 251-269.

Landreville, P. (1991). Le droit pénal de la route; commentaire du rapport de Clive Emsley. In Ph. Robert (Dir.), La Création de la loi et ses acteurs; l'exemple du droit pénal (185-193). Oñati: International Institute for the Sociology of Law.

Landreville, P. (1999). La surveillance électronique des délinquants: un marché en expansion. Déviance et Société, 23 (1), 105-121.

Landreville, P. (2002). Va-t-on vers une américanisation des politiques de sécurité en Europe?. In L. Mucchielli \& Ph. Robert (Dir.), Crime \& Sécurité, L'état des savoirs (424-433). Paris: La Découverte. 
Landreville, P. (2006). Ordre social et répression pénal: un demi-siècle de transformation. In R. Levy, L. Mucchielli \& R. Zauberman (Dir.), Crime et insécurité; un demi-siècle de bouleversements (289-313). Paris: L'Harmattan.

Landreville, P., Parent, C., Thomas, M. A., Fortier, J. C., Lafrance, G., Corte, E., \& Henri, N. (1986). Rapport du Comité d'étude sur les solutions de rechange à l'incarcération. Québec: Gouvernement du Québec, ministère du Solliciteur général.

Le Roy, E., \& Trotha, T. V. (Dir.). (1993). La violence et l'État; formes et évolution d'un monopole. Textes rassemblés et présentés par J. Lombard. Paris: L'Harmattan.

Lebeuf, M. E., \& Landreville, P. (1988). Analyse de la production des normes pénales du code criminel canadien dans le domaine de la circulation routière. Montréal: Université de Montréal, CICC.

Mauss, M. (1896). La religion et les origines du droit pénal d'après un livre récent. Revue d'bistoire des religions, 34, 269-295 et 35, 31-60.

Pires, A. P., \& Landreville, P. (1985) Les recherches sur les sentences et le culte de la loi. L'Année sociologique, 35, 83-113.

Pires, A. P., Landreville, P., \& Blankevoot, V. (1981). Système pénal et trajectoire sociale. Déviance et Société, 5 (4), 319-345.

Radbruch, G. (1950). Der Ursprung des Strafrechts aus dem Stande der Unfreien. In Radbruch, G. (Dir.), Elegantiae juris criminali. Basel: Verlag für Recht und Gesellschaft.

Radzinowicz, L. (1999). Adventures in Criminology. London: Routledge.

Robert, Ph. (Dir.). (1991). La Création de la loi et ses acteurs; l'exemple du droit pénal. Oñati: International Institute for the Sociology of Law.

Robert, Ph. (1996). La vengeance, l'État et la peine. Revue canadienne Droit et Société, 11 (2), 235-256.

Robert, Ph. (1998). Le monopole pénal de l'État. Esprit, 12, 134-153.

Robert, Ph. (1999). Le Citoyen, le crime et l'État. Genève-Paris: Droz.

Robert, Ph. (2000). Les territoires du contrôle social, quels changements. Déviance et Société, 24 (3), 215-235.

Robert, Ph. (2002). L'Insécurité en France. Paris: La Découverte.

Robert, Ph. (2005). Sociologie du crime. Paris: La Découverte.

Rousseaux, X., \& Levy, R. (Dir.). (1997). Le pénal dans tous ses États: Justice, États et sociétés en Europe (XIIème-XXème siècles). Bruxelles: Facultés universitaires Saint-Louis.

Rusche, G., \& Kirchheimer, O. (1939). Punishment and Social Structure. New York: Columbia University Press; tr. fr. Peine et structure sociale. Paris: Cerf, présenté et établi par R. Lévy et H. Zander, 1994.

Sellin, T. (1976). Slavery and the Penal System. New York: Elsevier.

Weber, M. (1986). Sociologie du droit. Paris: PUF, tr. fr. de RechtSoziologie rédigée entre 1911 et 1913.

Young, J. (1999). The Exclusive Society, Social Exclusion, Crime and Difference in Late Modernity. London: Sage. 